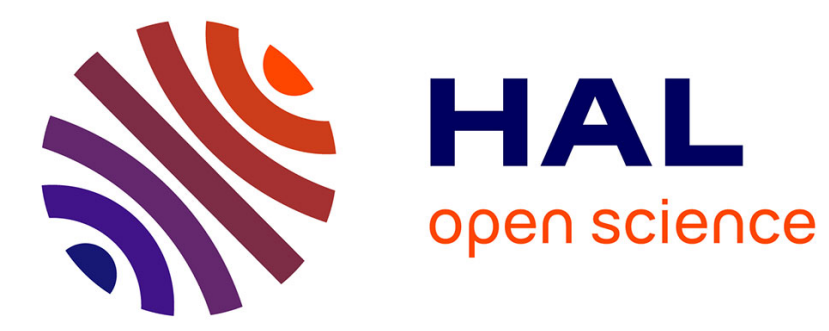

\title{
Electric discharge pumping of excimer lasers
}

\author{
W. Long, M. Bhaumik
}

\section{To cite this version:}

W. Long, M. Bhaumik. Electric discharge pumping of excimer lasers. Journal de Physique Colloques, 1979, 40 (C7), pp.C7-127-C7-140. 10.1051/jphyscol:19797435 . jpa-00219438

\section{HAL Id: jpa-00219438 https://hal.science/jpa-00219438}

Submitted on 1 Jan 1979

HAL is a multi-disciplinary open access archive for the deposit and dissemination of scientific research documents, whether they are published or not. The documents may come from teaching and research institutions in France or abroad, or from public or private research centers.
L'archive ouverte pluridisciplinaire HAL, est destinée au dépôt et à la diffusion de documents scientifiques de niveau recherche, publiés ou non, émanant des établissements d'enseignement et de recherche français ou étrangers, des laboratoires publics ou privés. 


\title{
Electric discharge pumping of excimer lasers $\left(^{*}\right)$
}

\author{
W. H. Long Jr. and M. L. Bhaumik \\ Northrop Corporation, Northrop Research and Technology Center, \\ Palos Verdes Penınsula, Calıfornıa 90274, U.S.A.
}

\begin{abstract}
Résumé. - Nous avons examiné le rendement et l'agrandissement à l'échelle des lasers à excimer (excités par de faisceau permet d'obtenir de haute énergie spécifique dans de grandes structures avec des courants de faisceau maintenue par faisceau électronique semble le plus prometteur pour l'obtention de grandes énergies. Les performances de ce type de laser peuvent être prédites grâce à un modèle englobant l'analyse de la cinétique des électrons, de la chimie de la décharge et le résonateur optique. Les limitations introduites par les problèmes de stabilité de décharge sont minimisées grâce à l'emploi d'un faisceau électronique uniforme. Cette bonne uniformité de faisceau permet d'obtenir de haute énergie spécifique dans de grandes structures avec des courants de faisceau électronique modérés.
\end{abstract}

\begin{abstract}
The efficiency and scalability of excimer lasers pumped by electric discharge is examined using the $\mathrm{KrF}$ laser as a generic example. E-beam sustained discharges show the greatest promise for scaling to high energy. Analyses of electron kinetics, discharge chemistry, and optical resonator are incorporated into a model for predicting the performance of such lasers. Limitations imposed by discharge stability are minimized by ensuring uniform e-beam deposition. With good uniformity, high specific energy can be achieved in large devices using low beam currents.
\end{abstract}

1. Introduction. - Excimer lasers currently provide the most efficient and powerful sources of coherent radiation in the ultraviolet and visible regions. These lasers have opened up the possibility of many important applications requiring visible and shorter wavelength lasers. Although significant progress has been made in developing these lasers, some crucial problems remain to be solved before their full potential can be realized. Efficient power transfer from the primary source of excitation to the laser medium is one of these problems and constitutes the major part of the discussion presented here.

An excimer is a molecule which exists only in an excited electronic state. The ground state is either repulsive or weakly bound at room temperature. The excited state is coupled to the ground state through a radiative transition typically in the visible or ultraviolet spectral regions. Lasers based on excimer transitions are free from the bottlenecking problem common to infrared and ion lasers, since the lower laser level is naturally depopulated in a very short time. Rare gases and some metal vapors form diatomic excimers in various configurations. These include homonuclear excimers, such as $\mathrm{Xe}_{2}^{*}$ and $\mathrm{Hg}_{2}^{*}$ and heteronuclear excimers (or exciplexes) such as $\mathrm{TlXe}^{*}$ and $\mathrm{CdHg}^{*}$. The rare gases also form excimers in association with oxygen and with the halogens.

(*) Work supported in part by the Advanced Research Projects Agency of the Department of Defense and monitored by the Office of Naval Research.
The rare gas oxides are characterized by long radiation lifetimes $\left(\sim 10^{-5} \mathrm{~s}\right)$ and small stimulated emission cross sections $\left(\sim 10^{-18} \mathrm{~cm}^{2}\right)$ which allow efficient excitation at low power densities; however, quenching of excited states and background absorption make the rare gas oxide lasers very inefficient. Most other excimers, however, have relatively short radiative lifetimes $\left(\sim 10^{-8} \mathrm{~s}\right)$ and large stimulated emission cross sections $\left(\sim 10^{16} \mathrm{~cm}^{2}\right)$. As a result, they require excitation at very high power densities in order to overcome fluorescence and quenching losses. Early researchers employed high current density electron beams and fast high pressure gas discharges to study these molecules.

The rare-gas dimers were the first excimers to demonstrate laser oscillations. They operate in the vacuum ultraviolet region of the spectrum from $126 \mathrm{~nm}$ to $176 \mathrm{~nm}$. Although the conversion of electrical energy into ions and excited states and the subsequent channeling into excimers is an extremely efficient process in the rare gases, the laser efficiency of these devices is severely limited by photoionization and electron deexcitation. The excited dimer of mercury, which was the first excimer to be observed in emission, has never been made to lase, apparently due to strong, broadband absorption from the lowest lying excited state.

The most efficient excimer lasers to date have been the rare gas halides which operate at wavelengths from $175 \mathrm{~nm}$ to $483 \mathrm{~nm}$. Their success is due in large part to the near unity branching ratio for conversion of rare gas ions and excited states into the excimer. 
In addition, absorption from the lowest lying excimer states is not significant. The principal limitation comes from other absorbers present in the medium, such as diatomic rare gas ions which limit the extraction efficiency to about fifty percent. Even so, the rare gas halides have so far demonstrated the highest efficiency and energy extraction per unit volume of any other laser in the visible or ultraviolet.

A typical potential energy diagram for the rare gas halide excimer is shown in figure 1. Formation of the excimer can proceed either through a long range Coulomb attraction between the positive rare gas ion and the negative halogen ion or along a curve crossing involving charge exchange between an excited rare gas atom and the neutral halogen. These formation paths, which take place via dissociative reactions or stabilized three body collisions, are referred to as the ion and metastable channels, respectively. The initial generation of rare gas ions and metastables has traditionally been accomplished with high energy electron beams. The efficiency for this process in argon is about $75 \%$ based on energy deposited in the gas. This number excludes energy dissipated in the pulse-forming network, the diode, and the foil and foil supports, as well as energy lost by electron transport out of the excited region. In an optimum laser mixture of argon, krypton and fluorine, roughly $80 \%$ of the argon ions and metastables form krypton fluoride $(\mathrm{KrF})$ excimers. When one accounts for the thermal energy lost in the formation process, only $20 \%$ of the deposited energy eventually resides in $\mathrm{KrF}$. With a $50 \%$ extraction efficiency limited by absorption, the laser efficiency of $\mathrm{KrF}$ becomes typically $10 \%$.

The efficiency of $\mathrm{KrF}$ and other rare gas halides can be improved by better electrical power condi-

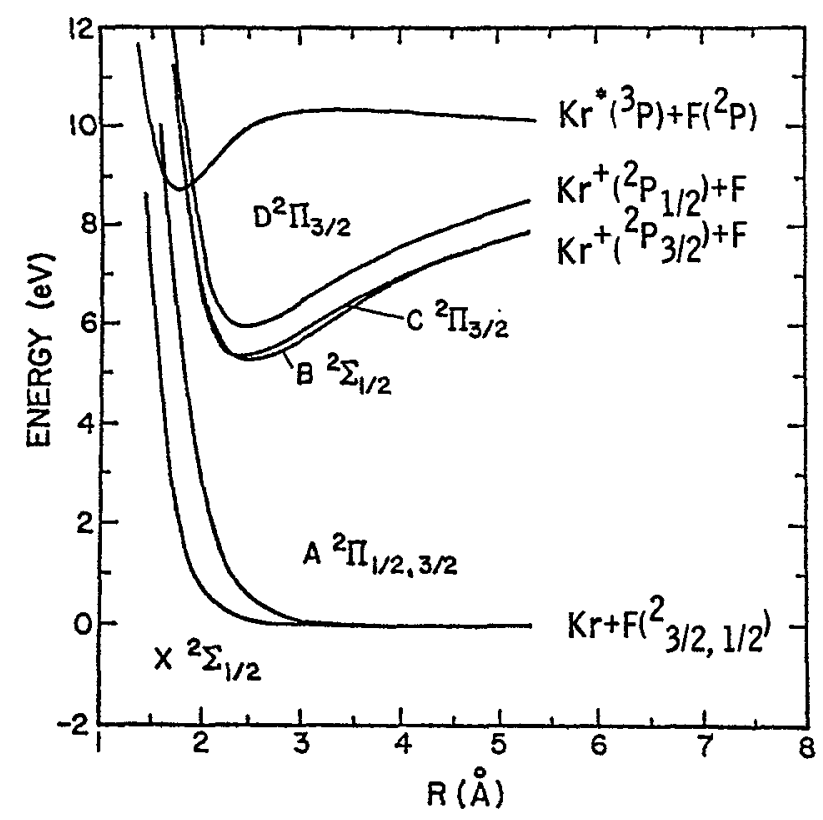

Fig. 1. - Potential energy curves for KrF. tioning, higher quantum efficiency, and reduced absorption. Improvements in power conditioning efficiency may be achieved through better circuit design to reduce internal losses, optimized coupling to the load, and recycling unused energy for subsequent pulses. Improved quantum efficiency can be achieved either by altering the ratio of power transferred to metastables as compared to ions or through the use of heavier rare gases with lower excitation and ionization energies. In high pressure rare gases pumped by relativistic electron beams, more than three times as many ions are created as metastables. If the same fraction of incident energy were to go exclusively into metastable states, the quantum efficiency would be improved by about $10 \%$. In the electric discharge, however, more metastables are produced than ions, since excitation takes place by accelerating electrons from low energy instead of decelerating them from high energy. In addition to improving the quantum efficiency, the reduced production of ions should result in less absorption. Therefore, discharge pumping of high pressure excimers is more desirable and will be discussed in further detail. Many approaches have been taken in applying electric discharge excitation to excimer lasers. They can be divided, however, into two basic groups : self-sustained and externally-sustained. In the selfsustained glow discharge, ionization is provided by electron avalanche in an applied field. This process is difficult to control once initiated and requires extremely uniform conditions to prevent collapse into an arc. Initial uniformity is achieved by carefully shaping the electrodes to produce a constant electric field and by preionizing the gas before the field is applied. If this were not done, avalanching would proceed from localized irregularities on the electrodes, sending the discharge directly into the arc phase. In the externally-sustained discharge, ionization is controlled by an external source such as an electron beam. This has the effect of decoupling the discharge impedance from the applied voltage. In the ideal case, the impedance is determined only by the gas density and the electron beam current density. The electric field can then be varied to optimize excitation of the desired species. In practice, the process of two-step ionization can transform the externallysustained discharge into a self-sustained one. This can be prevented only by uniform e-beam deposition and tailoring of the electric field or e-beam current density.

In the self-sustained discharge, excitation and laser energy extraction must be accomplished quickly before the discharge impedance falls to such a low value that the laser medium can no longer absorb power. During this impedance collapse, the electric field falls from a value above the breakdown field to a value where all the power dissipated in the discharge goes into gas heating. Thus, there is only a short period of time when the desired levels are 
efficiently excited. Strong coupling with the external circuit at such a low load impedance requires a low driving inductance which is difficult to achieve in large devices. An externally-sustained discharge, however, should have constant impedance as long as the external source is held constant. These devices then have the potential for long-pulse, high-energy operation. In fact, with the discharge increasing the deposited energy density, the volumetric efficiency should be much greater than with e-beam pumping alone. A KrF laser pumped by an e-beam sustained discharge has already demonstrated [1] $29 \mathrm{~J} / 1$ extracted energy from 0.121 at $11 \%$ intrinsic efficiency. Performance in larger devices has been much worse due to discharge instabilities caused by nonuniform ebeam deposition. These problems are discussed below using $\mathrm{KrF}$ as an example.

2. Electron kinetics in the $\mathrm{KrF}$ laser. - Since discharge pumping of the $\mathrm{KrF}$ laser has been demonstrated [1] to be more efficient then e-beam pumping, it is desirable to load as much energy into the discharge as possible. An analysis of the limit of energy loading and the laser efficiency requires an understanding of the electron and excited-state kinetics in the discharge. If the ratio of metastable density to ground state neutral density, $m / N$, is $10^{-5}$ or less, then electron-metastable collisions have a negligible effect on the electron energy distribution [2] and the electron kinetics can be decoupled from the excitedstate chemistry model. This allows a parameter study to be made of electron transport and excitation processes in the $\mathrm{KrF}$ laser which can be used as input to a complete laser model including optical resonator and external circuit [3].

The following discussion will emphasize the importance of including collisions between electrons via a screened Coulomb potential in the Boltzmann analysis of the heavy rare gases. At fractional electron densities typical of e-beam sustained $\mathrm{KrF}$ laser discharges $\left(n / N=3 \times 10^{-6}-3 \times 10^{-5}\right)$ these collisions appreciably alter both the electron mobility and the rate constants for excitation and ionization. Because of the sensitivity of the high energy tail of the electron energy distribution (Fig. 2), the effect of electron-electron collisions is most significant for those processes with the highest threshold energies. The reason that electron-electron collisions are not important in modeling the $\mathrm{CO}$ and $\mathrm{CO}_{2}$ lasers is that the pumping occurs at much lower energies and typical values of $n / N$ are only $10^{-7}$. These collisions must be considered, however, in analyzing discharge stability because of the large effect on ionization rates.

The method employed in this study to solve the Boltzmann transport equation has been described previously [4]. The contribution to the collision term arising from Coulomb collisions between electrons is derived by Shkarofsky et al. [5]. The value of $\ln A$ is taken to be 10 , where $\Lambda$ is the ratio of Debye length

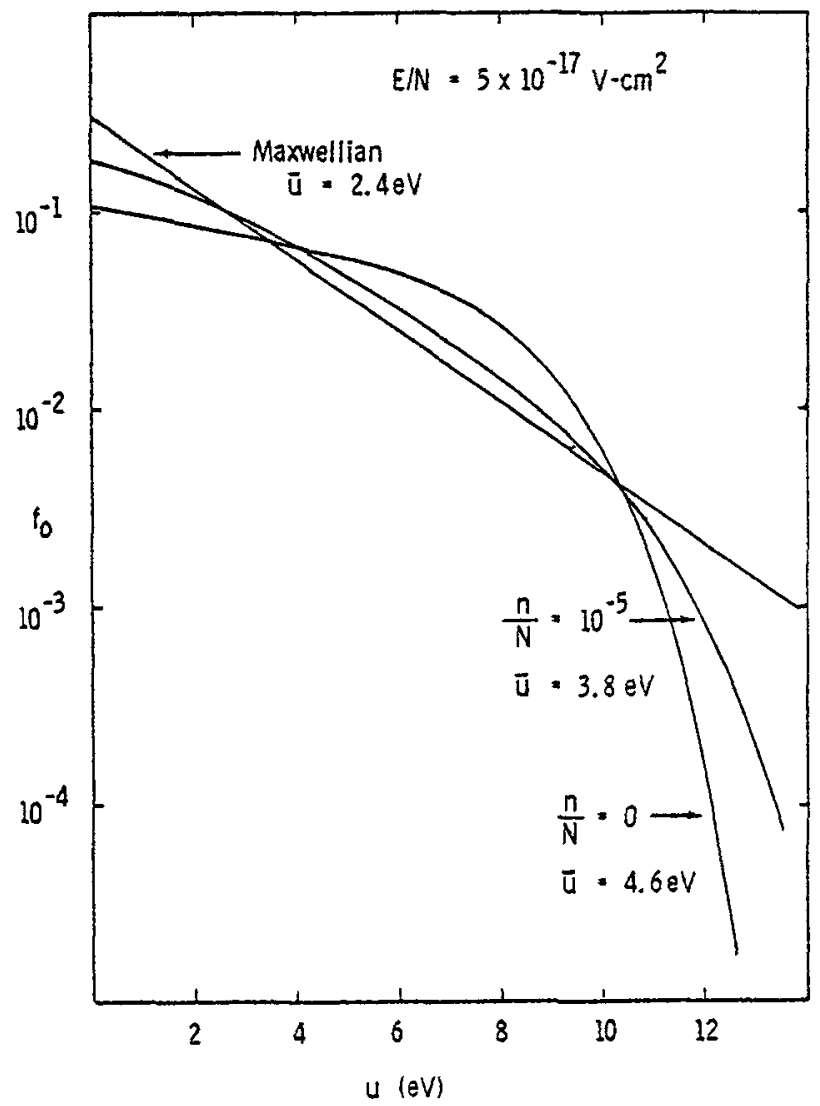

Fig. 2. - Electron energy distribution in Ar-Kr.

to the impact parameter for a $90^{\circ}$ deflection. The momentum transfer cross sections used are those of Milloy et al. [6] in argon and Frost and Phelps [7] in krypton. The cross sections for electronic excitation were measured by Schaper and Scheibner [8] and the normalization checked by Jacob et al. [9]. The data of Rapp et al. [10] were used for ionization of ground state atoms.

It is assumed that the effect of fluorine on the electron energy distribution is negligible for concentrations below a few tenths of one percent. The cross sections for direct vibrational and rotational excitation are expected to be small compared to dissociation [11]. A resonant process leads to negative ion production via dissociative attachment. The cross section for attachment of electrons to $F_{2}$ has recently been measured by Chantry [12]. Using this cross section, the effect of $F_{2}$ on the electron energy distribution was found to be small, except at low values of $E / N$ and $n / N$. Under normal operating conditions then, the attachment rate is proportional to the fluorine concentration and can be found from the measured cross section and the solutions of Boltzmann's equation in the rare gas mixture.

Because of the importance of electron-electron collisions, $E / N$ is no longer the sole parameter characterizing the electron energy distribution in a given 
gas mixture. The fractional ionization, $n / N$, must be specified as well. If the fractional metastable density, $m / N$, is less than $10^{-5}$, it is not an important parameter. Since the largest fractional metastable density consistent with stable discharge operation is about $3 \times 10^{-5}$, the effects of superelastic collisions and metastable ionization on the electron energy distribution can be neglected. In a stable discharge, therefore, we can completely characterize the electron properties in a given gas mixture with the two parameters, $E / N$ and $n / N$.

The electron drift velocity and mean energy are plotted in figure 3 as a function of $E / N$ for typical values of $n / N$ in a $95 \% \operatorname{Ar~} 5 \% \mathrm{Kr}$ mixture. The curves labeled $n / N=0$ apply to fractional ionizations below about $10^{-7}$. The drift velocity is found to be more than a factor of two higher at typical electron densities than it is at low electron density where electron-electron collisions are unimportant. The calculated drift velocity in pure argon with $n / N=0$ agrees within $5 \%$ with the experimental data of Pack et al. [13], Robertson [14] and Brambring [15] over the $E / N$ range from $10^{-19} \mathrm{~V} \mathrm{~cm}^{2}$ to $10^{-15} \mathrm{~V} \mathrm{~cm}^{2}$. The power loading into the discharge is given by

$$
E . J=e N^{2} \frac{n}{N} v_{\mathrm{e}}\left(\frac{E}{N}, \frac{n}{N}\right) \frac{E}{N} .
$$

As a result of the $n / N$ dependence of $v_{\mathrm{e}}$, this function will increase faster than linearly with $n / N$ at cons$\tan t E / N$.

All the energy which goes into the discharge is converted through various channels into heat, electronic excitation, or ionization of the gas. The dominant processes include heating by momentum transfer

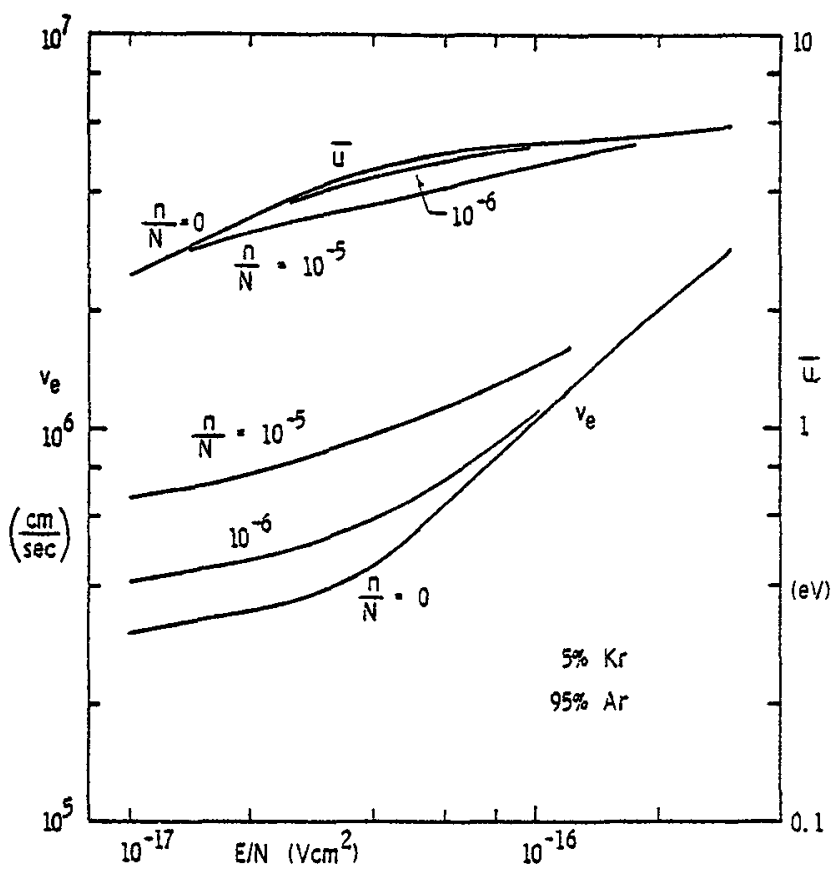

Fig. 3. - Electron drift velocity and mean energy.

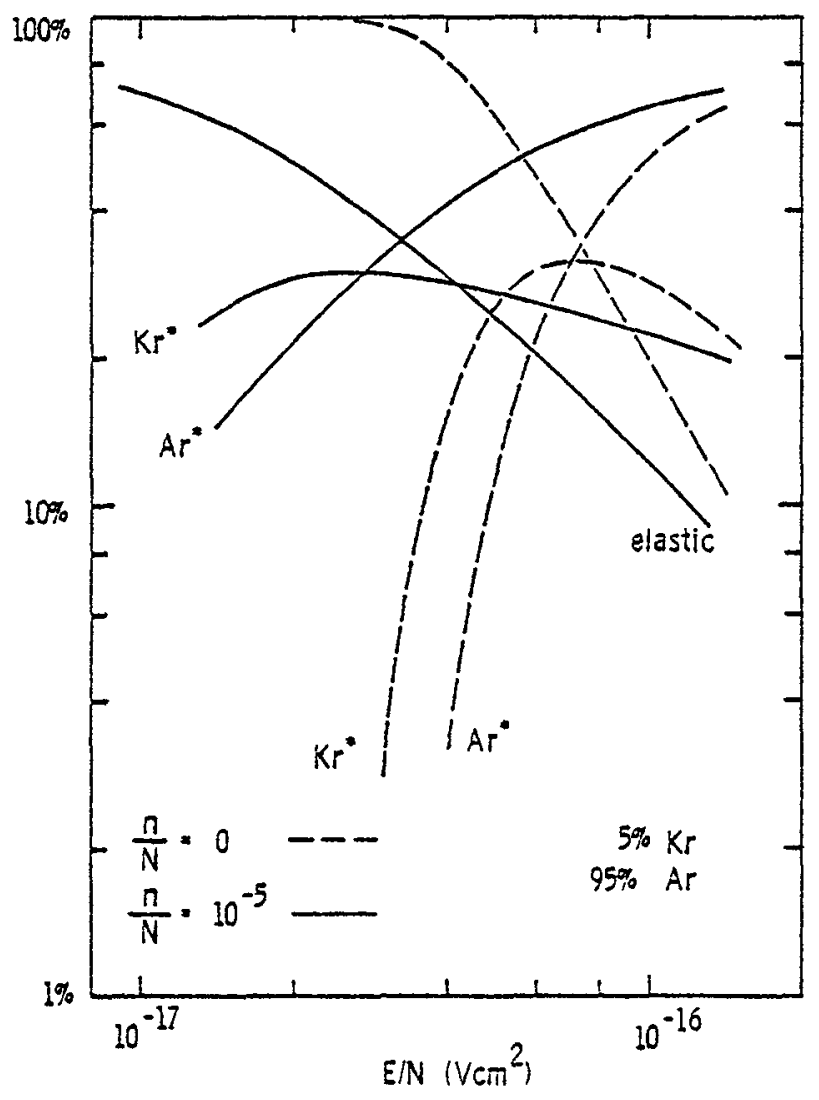

Fig. 4. - Discharge power partitioning.

in elastic collisions and excitation of various electronic states in argon and krypton. The cross sections for excitation of these electronic states are lumped together for each gas and represent an effective cross section for production of the metastable. The fractional power into each of these channels is plotted in figure 4 as a function of $E / N$ for typical values of $n / N$. The power going into ionization of metastables and ground state atoms is negligible in the parameter range considered here. The effect of electron-electron collisions is dramatic in moving the range of efficient production of metastables to lower values of $E / N$. This has a significant impact on the predicted operating regime of the $\mathrm{KrF}$ laser.

The highest specific laser energy to date from an e-beam sustained discharge in $\mathrm{Ar}-\mathrm{Kr}-\mathrm{F}_{2}$ was observed at an $E / N=3 \times 10^{-17} \mathrm{~V} \mathrm{~cm}^{2}$ [1]. The measured discharge efficiency was $20 \%$, and yet without electron-electron collisions the theory would predict virtually no pumping of metastable states by the discharge at this $E / N$.

The quantum efficiency for producing $\mathrm{KrF}^{*}$ can be derived from the power partitioning curves by defining

$$
\eta=\frac{h v}{e}\left[f\left(\mathrm{Ar}^{*}\right) / 11.8 \mathrm{eV}+f\left(\mathrm{Kr}^{*}\right) / 9.9 \mathrm{eV}\right] .
$$

The numerator in each term is the fraction of discharge power going into the metastable and the denominator 


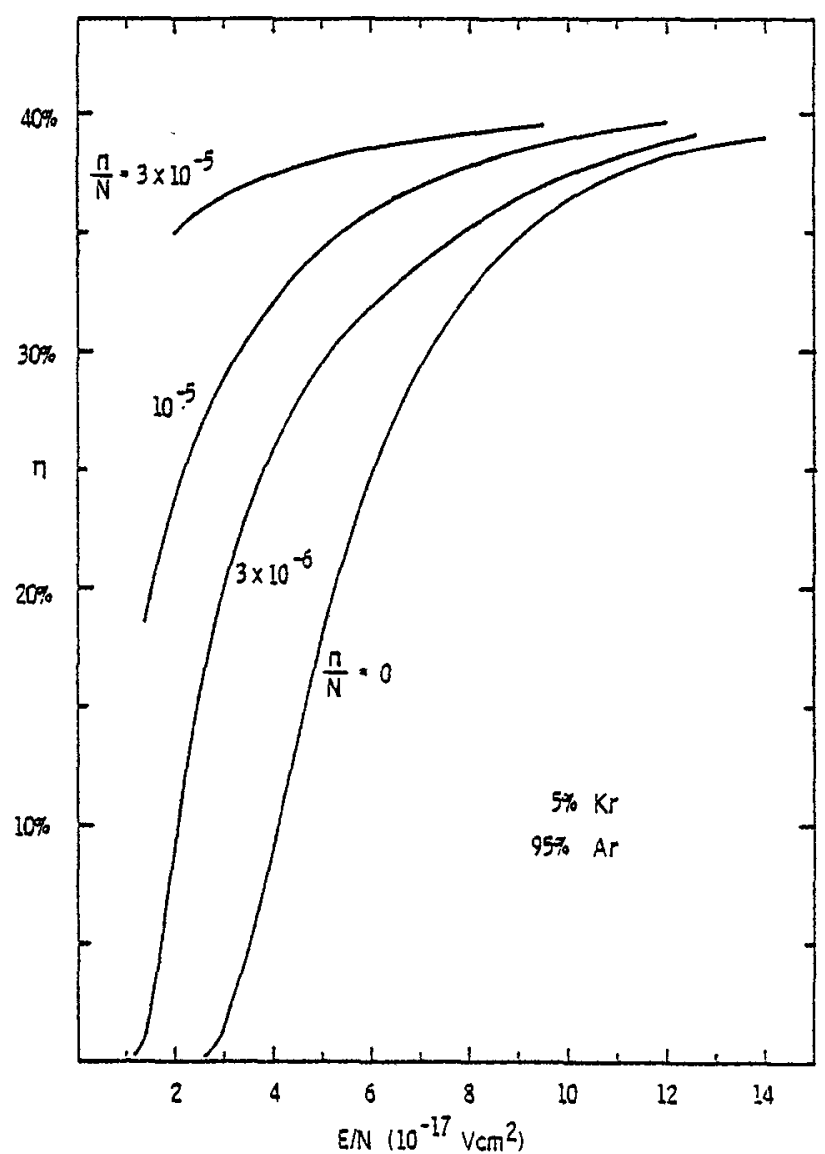

Fig. 5. - Quantum efficiency for producıng $\mathrm{KrF}^{*}$.

is the energy of the metastable. This function is plotted in figure 5. At low $E / N$ the efficiency falls off because of elastic heating and at high $E / N$ it approaches the quantum efficiency of $\mathrm{Ar}^{*}$ at $42 \%$. The actual production efficiency will be slightly less than this because the excitation is distributed over a number of levels above the metastable. This correction will be least important at low $E / N$ where the electrons excite preferentially those states with the lowest thresholds. Reactions which intercept the energy transfer from the metastables to $\mathrm{KrF}^{*}$ will also reduce the production efficiency.

The maximum extraction efficiency for converting these upper laser levels to laser photons is given approximately by $\frac{t}{a+t}$, where $t$ is output coupling and $a$ is the round trip cavity absorption. For the device in reference 1 with a $50 \mathrm{~cm}$ cavity containing 4 torr $\mathrm{F}_{2}, a \approx 20 \%$. With $t=20 \%$, the maximum extraction efficiency is $50 \%$. From figure 5 the discharge efficiency at $E / N=3 \times 10^{-17} \mathrm{~V} \mathrm{~cm}^{2}$ and $n / N=3 \times 10^{-5}$ is $36 \%$. The resulting net efficiency based on energy deposited in the discharge is therefore $18 \%$ in good agreement with experiment. The efficiency would appear to be a strong function of $E / N$ and $n / N$. However, operating conditions in an e-beam sustained discharge are such that high electron densities give rise to low electric field strengths and consequently the discharge efficiency is nearly constant.

In summary, a parametric study of the electron kinetics in an $\mathrm{Ar}-\mathrm{Kr}-\mathrm{F}_{2}$ discharge shows that $E / N$ and $n / N$ are the critical parameters, while $m / N$ and $\mathrm{F}_{2} / N$ can be neglected when they are smaller than $10^{-5}$ and $10^{-3}$ respectively. Electron-electron collisions play a significant role in increasing electron mobility and in raising the metastable production efficiency at low values of $E / N$. Any comprehensive model of the rare gas halide lasers must include these effects.

3. KrF kinetics. - The kinetics which lead to the population of the $\mathrm{KrF}^{*}$ state in an $\mathrm{Ar}-\mathrm{Kr}-\mathrm{F}_{2}$ mixture have been investigated by various laboratories. The principal channels under moderate e-beam pumping $\left(<50 \mathrm{~A} / \mathrm{cm}^{2}\right)$ proceed through the argon ion and metastable states. At high current densities, the production of $\mathrm{Ar}^{+}$and $\mathrm{Ar}^{*}$ under e-beam excitation is roughly 3.5 to 1 with a total efficiency of $75 \%$. The remaining $25 \%$ of the incident energy is lost via radiation and elastic heating. At current densities less than $50 \mathrm{~A} / \mathrm{cm}^{2}$ the principal neutralization process is ion-ion recombination, i.e.

$$
\begin{aligned}
& \mathrm{Kr}^{+}+\mathrm{F}^{-}+\mathrm{M} \rightarrow \mathrm{KrF}^{*}+\mathrm{M} \\
& \mathrm{Kr}_{2}^{+}+\mathrm{F}^{-} \longrightarrow \mathrm{KrF}^{*}+\mathrm{Kr} .
\end{aligned}
$$

These reactions are responsible for populating the $\mathrm{KrF}^{*}$ and $\mathrm{ArF}^{*}$ states. Some of the $\mathrm{ArF}^{*}$, which is formed in the recombination of $\mathrm{Ar}^{+}$and $\mathrm{Ar}_{2}^{+}$, is converted to $\mathrm{KrF}^{*}$ through the displacement reaction,

$$
\mathrm{ArF}^{*}+\mathrm{Kr} \rightarrow \mathrm{KrF}^{*}+\mathrm{Ar} .
$$

The rest is lost via quenching and spontaneous emission. This loss amounts to about $10 \%$ of the $\mathrm{Ar}^{+}$ ions originally formed. Since it takes about $26 \mathrm{eV}$ to create each $\mathrm{Ar}^{+}$ion, the quantum efficiency for producing $\mathrm{KrF}^{*}$ at $5 \mathrm{eV}$ from $\mathrm{Ar}^{+}$is $19 \%$, and the net efficiency of the ion channel is then $17 \%$. This, however, neglects the metastables which are also formed by e-beam excitation. The same $26 \mathrm{eV}$ which produced one ion will also generate about 0.3 metastables which have a $63 \%$ chance of forming $\mathrm{KrF}^{*}$. This raises the overall efficiency for e-beam pumping to $20 \%$. The neutral channel processes will be discussed below.

Under discharge pumping of $\mathrm{KrF}$, about $80 \%$ of the input power goes into excited rare gas states $\mathrm{Ar}^{*}$ and $\mathrm{Kr}^{*}$. The remaining $20 \%$ is lost in elastic scattering and dissociative attachment to $F_{2}$. The ratio of $\mathrm{Ar}^{*}$ to $\mathrm{Kr}^{*}$ produced is about two to one as shown in figure 4. Very few ions are generated by the discharge. There are two dominant channels for converting $\mathrm{Ar}^{*}$ to $\mathrm{KrF}^{*}$ (Fig. 6) ; one through the $\mathrm{ArF}^{*}$ state and the other through the $\mathrm{Kr}^{*}$ state. Typically, with a total pressure of three atmospheres and 


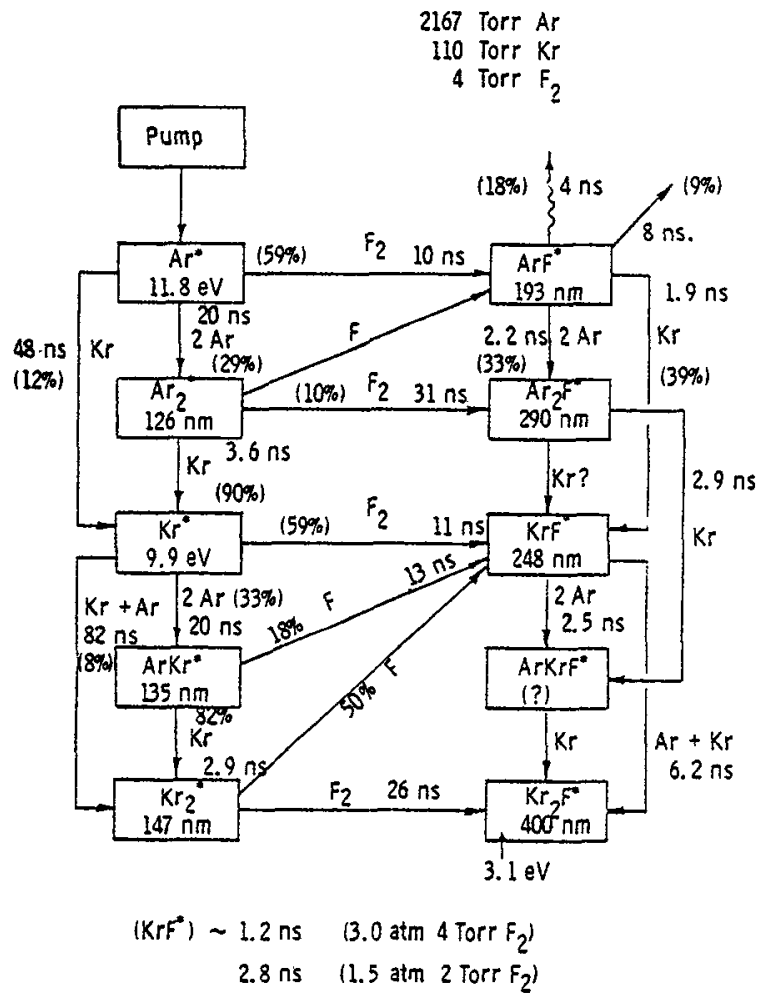

F1g. 6. - Neutral channels.

$0.17 \% \mathrm{~F}_{2}$, about $60 \%$ of the $\mathrm{Ar}^{*}$ react with $\mathrm{F}_{2}$ to form $\mathrm{ArF}^{*}$, which has a $55 \%$ chance of being converted to $\mathrm{KrF}^{*}$. About $37 \%$ of the $\mathrm{Ar}^{*}$ undergo excitation transfer to $\mathrm{Kr}^{*}$, either directly or via $\mathrm{Ar}_{2}^{*}$. The remaining $3 \%$ end up as $\mathrm{Ar}_{2} \mathrm{~F}^{*}$. The $\mathrm{Kr}^{*}$, formed either through this channel or directly by the discharge, have a $60 \%$ chance of producing $\mathrm{KrF}^{*}$. The other $40 \%$ go into forming the rare gas dimers ArKr* and $\mathrm{Kr}_{2}^{*}$. It is believed that when these excited states react with $\mathrm{F}_{2}$ about half of them produce $\mathrm{KrF}^{*}$ and the rest form the $\mathrm{ArKrF}^{*}$ and $\mathrm{Kr}_{2} \mathrm{~F}^{*}$ states. The resulting discharge efficiency is summarized below :

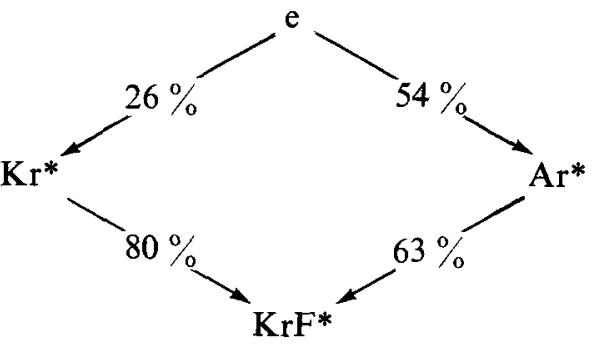

Quantum

efficiency

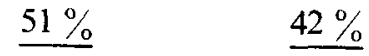

Net

$10.6 \%$

$14.3 \%$
Thus discharge pumping of $\mathrm{KrF}^{*}$ is slightly more efficient than pure e-beam pumping based on energy deposited. However, wall-plug efficiency for the e-beam device will be much less due to a $50 \%$ loss in the cooled foil and foil support structure and additional losses due to scattering in the gas. The e-beam also produces molecular ions which absorb at the laser frequency. In a practical device it is therefore desirable to achieve the highest possible ratio between discharge and e-beam pumping consistent with long pulse stability.

The production rate of the $\mathrm{KrF}^{*}$ state, $R_{p}$, can now be expressed as a function of the input power. Thus,

$$
R_{p} h v=0.25 P_{d}+0.20 P_{e b}
$$

where $P_{d}$ and $P_{e b}$ are the discharge and e-beam power absorbed by the gas. The loss of $\mathrm{KrF}^{*}$ includes quenching by $F_{2}$, spontaneous emission and a threebody reaction leading to $\mathrm{Kr}_{2} \mathrm{~F}^{*}$. All of these processes will be lumped into a single decay rate, $1 / \tau$. If the gain profile is homogeneously broadened, then the loss of $\mathrm{KrF}^{*}$ by stimulated emission is proportional to the laser intensity, $I$. The continuity equation for $\mathrm{KrF}^{*}$ is then

$$
\frac{\partial N^{*}}{\partial t}=R_{p}-N^{*} / \tau-\sigma I N^{*} / h v
$$

where $\sigma$ is the cross section for stimulated emission.

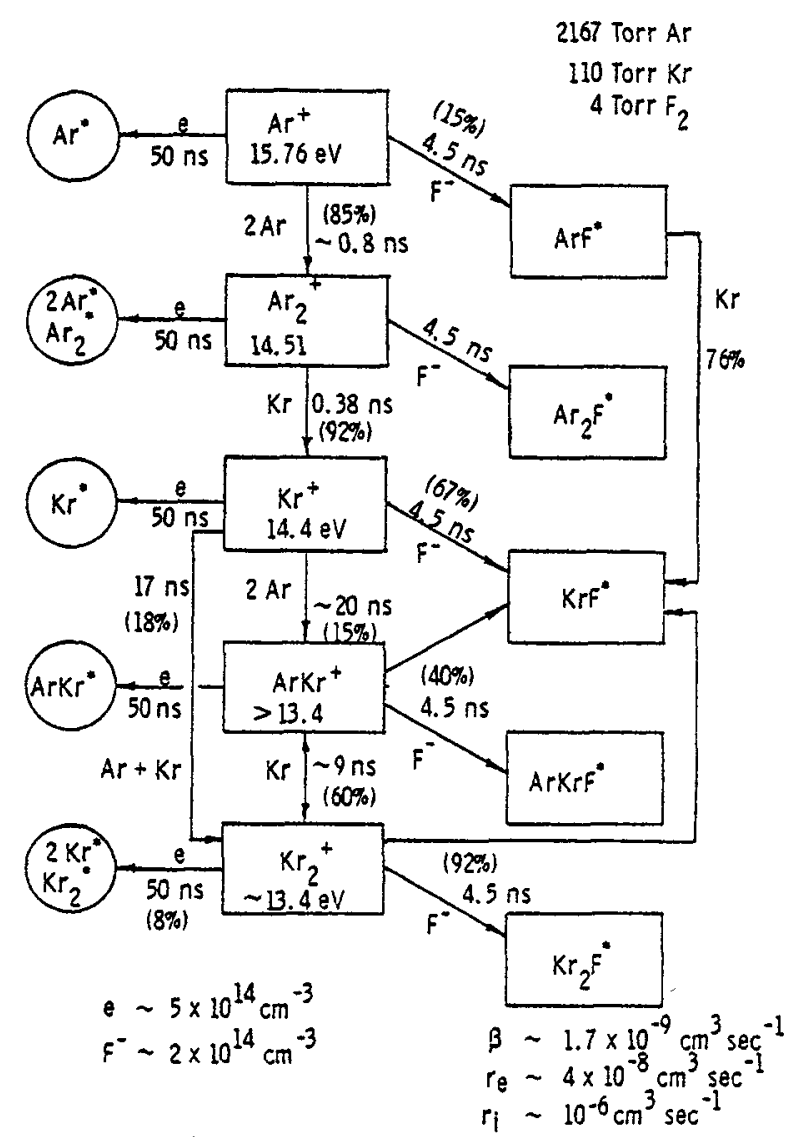

Fig. 7. - Ion channels. 
The steady state population density is given by

$$
N^{*}=R_{p} /(1 / \tau+\sigma I / h v) .
$$

In terms of the saturated gain, $g=\sigma N^{*}$, and the small signal gain, $g_{0}=R_{p} \sigma \tau$, this becomes

$$
g=g_{0}\left(1+I / I_{s}\right) \text {. }
$$

The laser saturation intensity, $h v / \sigma \tau$, is the intensity at which stimulated emission equals quenching.

4. Optical resonator. - As discussed above, for the case of homogeneous laser transitions, the saturated gain coefficient $g$ is related to the small signal gain coefficient $g_{0}$ by $g=g_{0} /\left(1+\xi_{+}+\xi_{-}\right)$, where $\xi_{+}$and $\xi_{-}$are the laser intensities for the $+x$ and $-x$ directions normalized to the saturation intensity. In addition to gain, the $\mathrm{KrF}$ laser medium contains several species which absorb at the laser frequency. This absorption is distributed throughout the cavity and may also show saturation effects. The species which exhibit gain and absorption in the $\mathrm{KrF}$ laser are listed in table I along with their respective saturation intensities defined as $I_{s}=h v / \sigma \tau$ where $\sigma$ is the optical cross section for stimulated emission or absorption and $\tau$ is the mean lifetime of the particle.

\begin{tabular}{|c|c|c|c|c|}
\hline & $\sigma\left(\AA^{2}\right)$ & $\tau(\mathrm{ns})$ & $I_{\mathrm{s}}\left(\mathrm{MW} / \mathrm{cm}^{2}\right)$ & \\
\hline & - & - & - & \\
\hline $\mathrm{KrF}^{*}$ & 2 & 1.5 & 2.6 & $3 \mathrm{~atm}$ \\
\hline $\mathrm{Ar}_{2}^{+}$ & 0.15 & 2.8 & 19 & $5 \% \mathrm{Kr}$ \\
\hline $\mathrm{Kr}_{2}^{+}$ & 0.075 & 2.8 & 38 & $0.17 \% \mathrm{~F}_{2}$ \\
\hline $\mathrm{F}^{-}$ & 0.085 & 2 & 47 & $20 \mathrm{~A} / \mathrm{cm}^{2}$ \\
\hline $\mathrm{F}_{2}$ & 0.00015 & - & - & \\
\hline
\end{tabular}

Table I.

The growth of laser intensity in the $+x$ direction is governed by the equation,

$$
\frac{1}{\xi_{+}} \frac{\partial \xi_{+}}{\partial x}=\frac{g_{0}}{1+\xi_{+}+\xi_{-}}-\sum_{n} \frac{\alpha_{n}}{1+\left(\xi_{+}+\xi_{-}\right) \frac{I_{s}}{I_{n}}}
$$

where $I_{s}$ is the saturation intensity for stimulated emission and $I_{n}$ is the saturation intensity for the $n$th absorbing species. Since the gain and absorption are isotropic $\frac{1}{\xi_{+}} \frac{\partial \xi_{+}}{\partial x}=-\frac{1}{\xi_{-}} \frac{\partial \xi_{-}}{\partial x}$ and consequently

$$
\xi_{+} \xi_{-}=\text {constant }=\xi_{0} .
$$

Using this expression to relate $\xi_{-}$to $\xi_{+}$in eq. (3) we have, dropping the pluses,

$$
\frac{1}{\xi} \frac{\partial \xi}{\partial x}=\frac{g_{0}}{1+\xi+\xi_{0}^{2} / \xi}-\sum_{n} \frac{\alpha_{n}}{1+\left(\xi+\xi_{0}^{2} / \xi\right) \frac{I_{s}}{I_{n}}} .
$$

If the cavity is defined by a perfect reflector at $x=0$ and a partial with reflectivity $R$ at $x=L$, then

$$
\xi(0)=\xi_{0} \text { and } \xi(L)=\xi_{0} / \sqrt{R}
$$

When eq. (4) is integrated from $x=0$ to $x=L$, an expression relating $\xi_{0}, R, g_{0}$, and $\alpha_{n}$ is obtained in principle. Then the laser output intensity is given by $(1-R) \xi(L)=\xi_{0}(1-R) / \sqrt{R}$.

In practice a simple closed-form solution to eq. (4) is only obtained if we approximate the distributed absorption as a lumped loss, $a$, in the output mirror. Integrating eq. (4) without the absorption term we obtain,

$$
\left(\xi-\xi_{0}\right)+\ln \left(\xi / \xi_{0}\right)-\xi_{0}^{2}\left(\frac{1}{\xi}-\frac{1}{\xi_{0}}\right)=g_{0} x .
$$

Evaluating this at $x=L$ with the boundary condition $\xi(L)=\xi_{0} / \sqrt{R}$ gives,

$$
(1-R) \xi_{0} / \sqrt{R}=g_{0} L-\frac{1}{2} \ln \frac{1}{R} .
$$

The output intensity, defined as

$$
I_{\text {out }}=(1-R-a) \xi(L) I_{s},
$$

is then

$$
I_{\text {out }}=I_{s} \frac{t}{a+t}\left(g_{0} L-\frac{1}{2} \ln \frac{1}{R}\right)
$$

where $t=1-R-a$ is the transmissivity of the output mirror.

We now define the extraction efficiency, $\eta_{c}$, as the ratio of actual output intensity to the intensity which would be obtained if every $\mathrm{KrF}^{*}$ generated in the cavity resulted in a photon leaving the output mirror. This maximum intensity is $I_{\max }=R_{p} V h v / A=g_{0} L I_{s}$, where $R_{p}$ is the generation rate per unit volume of $\mathrm{KrF}^{*}$. The extraction efficiency from eq. (7) is then

$$
\eta_{c}=\frac{t}{a+t}\left(1-\frac{1}{g_{0} 2 L} \ln \frac{1}{R}\right)
$$

In the $\mathrm{KrF}$ laser, the approximation of lumped losses at the mirrors is not very good, since the round trip absorption, $a$, often approaches unity and the resulting transmissivity, $t$, may go negative. Furthermore, the threshold gain derived from eq. (8) is independent of the absorption, a result which is clearly unphysical if the absorption is distributed. For if gain equals absorption, the medium will certainly not lase even though eq. (8) says it will if we chose the right reflectivity.

An approximation which yields more viable results in the presence of large absorption losses is to assume that the intracavity intensity, $I=\left(\xi_{+}+\xi_{-}\right) I_{s}$, is constant, independent of $x$. Eq. (4) can now be integrated readily to obtain, $\ln \left(\xi / \xi_{0}\right)=(g-\alpha) x$, where $g$ and $\alpha$ are the saturated gain and absorption respectively. Applying the previous boundary condition at $x=L$ yields

$$
\frac{1}{2 L} \ln \frac{1}{R}=g-\alpha=\frac{g_{0}}{1+I / I_{s}}-\sum_{n} \frac{\alpha_{n}}{1+I / I_{n}} .
$$


After multiplying both sides by $c$, this equation says that the rate at which photons are being extracted from the cavity is equal to the difference between the rate at which they are produced and the rate at which they are absorbed. The output intensity is just the product of the extraction rate and the number of photons in the cavity divided by the area of the output aperture, $A$, i.e.

$$
I_{\text {out }}=\frac{c}{2 L} \ln \frac{1}{R} \cdot \frac{I V}{\operatorname{ch} v} \cdot \frac{1}{A} \cdot h v=\frac{I}{2} \ln \frac{1}{R} .
$$

If $I_{n} \gg I_{s}$ (see Table 1) then $\alpha \sim \sum_{n} \alpha_{n}$ and eq. can be solved for $I$ directly. Thus,

$$
I=I_{s}\left(\frac{g_{0}}{\alpha+\frac{1}{2 L} \ln \frac{1}{R}}-1\right)
$$

and the output intensity is therefore,

$$
I_{\text {out }}=\frac{I_{s}}{2}\left(\ln \frac{1}{R}\right)\left(\frac{g_{0}}{\alpha+\frac{1}{2 L} \ln \frac{1}{R}}-1\right) .
$$

The extraction efficiency analagous to eq. (8) is

$$
\eta_{c}=\frac{1}{2 L}\left(\ln \frac{1}{R}\right)\left(\frac{1}{\alpha+\frac{1}{2 L} \ln \frac{1}{R}}-\frac{1}{g_{0}}\right) \text {. }
$$

This expression has been compared with the numerical solution of eq. (4) and was found in good agreement for values of $R$ down to $30 \%$ independent of $\alpha$. In contrast to eq. (8) the threshold gain is now a function of the distributed absorption. If there is also some lumped absorption, $a$, in the optical components, this can be taken care of as before and the final expression for extraction efficiency becomes,

$\eta_{c}=\frac{t}{a+t} \frac{1}{2 L}\left(\ln \frac{1}{R}\right)\left(\frac{1}{x+\frac{1}{2 L} \ln \frac{1}{R}}-\frac{1}{g_{0}}\right)$.

If $a \ll t$ the optimum reflectivity is given by

$$
\frac{1}{2 L} \ln \frac{1}{R}=\sqrt{\alpha g_{0}}-\alpha
$$

and the maximum extraction efficiency is, from eq. (14),

$$
\eta_{c}^{\max } \sim\left(1-\sqrt{\frac{2}{y_{0}}}\right)^{2} .
$$

This function is plotted in figure 8 .

In a practical device the mirrors cannot be placed directly adjacent to the gain region. Because of the electric field applied to the discharge, the mirrors must be set back a distance at least equal to the electrode separation. Alternatively, windows may be

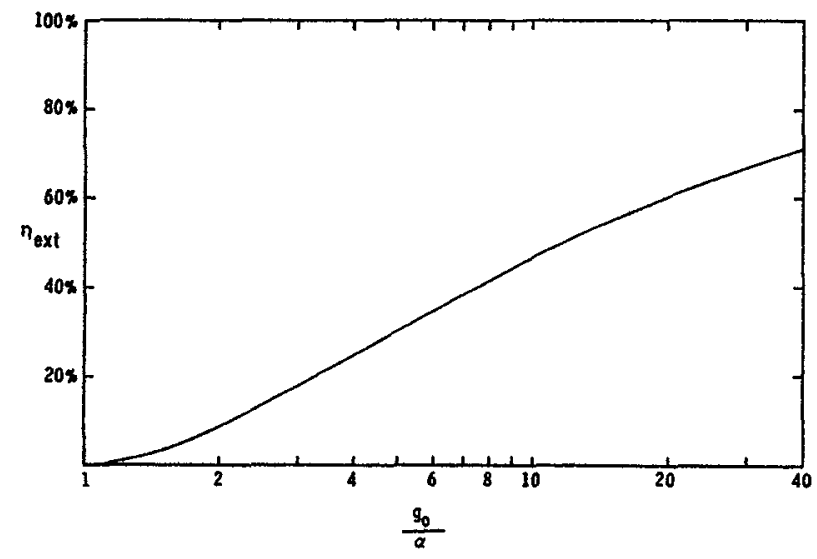

Fig. 8. - Laser extraction efficiency.

used to contain the laser gas and the mirrors mounted externally. We must then define several different lengths and modify our expression for the extraction efficiency and optimum reflectivity. Let $L$ remain the mirror separation and define $l_{s}$ as the gain length and $l_{n}$ the length containing the $n$th absorbing species. Then eq. (14) becomes

$\eta_{c}=\frac{t}{a+t} \frac{1}{2}\left(\ln \frac{1}{R}\right)\left(\frac{1}{\sum_{n} \alpha_{n} l_{n}+\frac{1}{2} \ln \frac{1}{R}}-\frac{1}{g_{0} l_{s}}\right)$

and the optimum reflectivity is,

$$
\frac{1}{2} \ln \frac{1}{R}=\sqrt{g_{0} l_{s} \sum_{n} \alpha_{n} l_{n}}-\sum_{n} \alpha_{n} l_{n} .
$$

5. Discharge stability. - The glow discharge, which is an effective excitation technique for a variety of gas lasers, is unstable at high pressures unless sustained by an external ionization source. In the selfsustained mode, the initial glow collapses into a filamentary arc after a time which depends on the pressure and discharge power density. This occurrence can be prevented by keeping the discharge in a regime where the principal ionization is supplied externally.

In the e-beam sustained $\mathrm{KrF}$ laser, a uniform glow can be maintained for times up to a microsecond if the discharge voltage is kept below a well-defined limit. Under heavy pumping conditions, the ionization of metastable states becomes a significant source of electrons. When this two-stage ionization exceeds ionization by the e-beam, the discharge goes into a volume runaway and arcs after a few hundred nanoseconds. In order to determine the limits of the stable operating regime, a simple $\mathrm{KrF}$ discharge model is proposed as set forth in figure 9.

The quantities $S_{i}$ and $S_{m}$ are the e-beam source terms for ions and metastables respectively. The metastable ionization rate, $k_{\text {mit }}$, is approximately equal to $6 \times 10^{-8} \mathrm{~cm}^{3} \mathrm{~s}^{-1} . \beta$ is the attachment rate 
Plasma kinetics .

$$
\begin{aligned}
\frac{\partial n}{\partial t} & =S_{i}+k_{m} m n-\beta F_{2} n \\
\frac{\partial F_{-}}{\partial t} & =\beta F_{2} n-\gamma F_{-}\left(n+F_{-}\right) \\
\frac{\partial m}{\partial t} & =S_{m}+k_{m} N n-k_{m i} m n-Q F_{2} M .
\end{aligned}
$$

Discharge circuit :

$$
\begin{gathered}
L \frac{\mathrm{d}^{2} I}{\mathrm{~d} t^{2}}+\frac{\mathrm{d} V}{\mathrm{~d} t}+I / C=0 \\
I=e A n v_{e}+\frac{V}{R} .
\end{gathered}
$$

Fig. 9. - Simple KrF laser model.

to fluorine and $\gamma$ is the ion-ion recombination rate. The continuity equations for the metastables can be lumped together with a single density, $m$, because the quenching rate, $Q$, and ionization rate, $k_{m i}$, are fairly independent of species. The metastable production rate, $k_{m}$, is then a weighted sum of the production rates for $\mathrm{Ar}^{*}$ and $\mathrm{Kr}^{*}$. This rate is a strong function of $E / N$ and is plotted in figure 10 for several values of fractional ionization $n / N$.

Since the time constant for the external circuit,

$$
\sqrt{L C} \sim 200-500 \mathrm{~ns}
$$

is typically large compared to that for equilibrium of the ion and metastable species densities, the equations for the latter can be solved in the quasisteady-state approximation. The stability of the

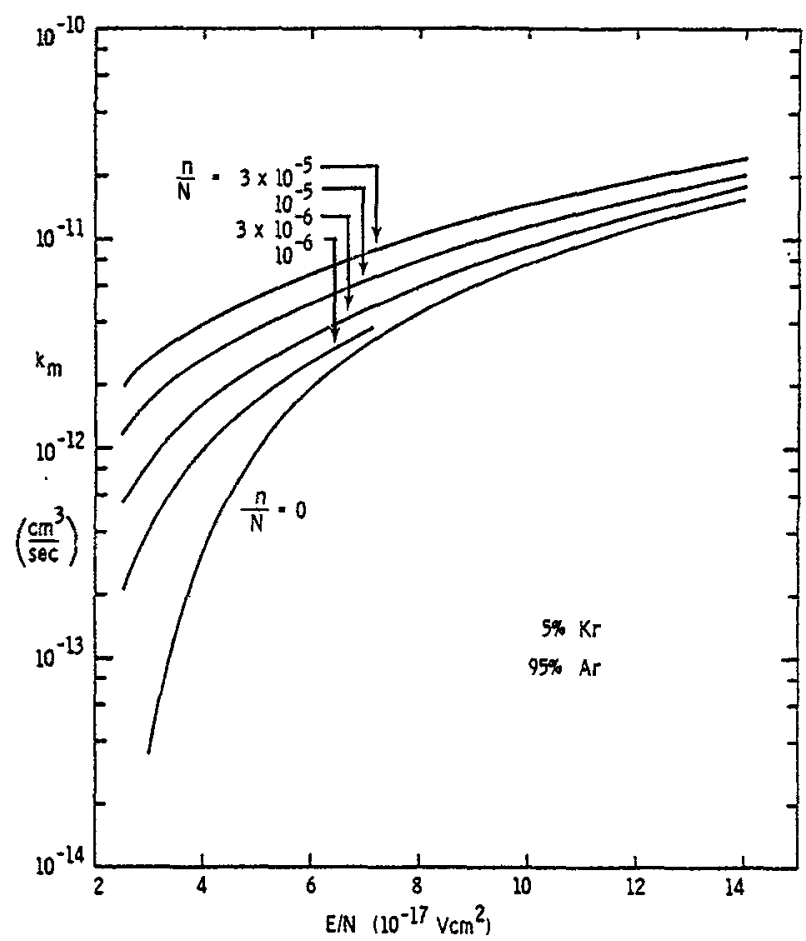

Fig. 10. - Metastable production rate. steady-state solution is then found by applying a small perturbation with time dependence proportional to $\exp (-i \omega t)$ and looking for positive imaginary eigenvalues of $\omega$. When this is done for the equations in figure 9 , the resulting linearized system for the perturbation on electron and metastable densities is,

$$
\begin{array}{r}
\left(\mathrm{i} \omega+k_{m i} m_{0}-\beta \mathrm{F}_{2}\right) \tilde{n}+k_{m i} n_{0} \tilde{m}=0 \\
\left(k_{m} N-k_{m i} m_{0}\right) \tilde{n}+\left(\mathrm{i} \omega-k_{m i} n_{0}-Q \mathrm{~F}_{2}\right) \tilde{m}=0 .
\end{array}
$$

The eigenvalues are then solutions of

$$
\begin{aligned}
\omega^{2}+\mathrm{i} \omega & \left(\frac{S_{i}}{n_{0}}+Q \mathrm{~F}_{2}+k_{m i} n_{0}\right)- \\
& -\frac{S_{i}}{n_{0}} Q \mathrm{~F}_{2}-k_{m i} n_{0}\left(\beta \mathrm{F}_{2}-k_{m} N\right)=0 .
\end{aligned}
$$

Thus $2 \omega=-b \pm \sqrt{b^{2}-4 c}$, where $b$ is the coefficient of $\omega$ in eq. (21) and $c$ is the constant term. Since $b$ is positive imaginary, $\omega$ will have a positive imaginary part only if $c>0$. The stability requirement is then,

$$
\frac{S_{i}}{n_{0}} Q \mathrm{~F}_{2}>k_{m i} n_{0}\left(k_{m} N-\mathrm{F}_{2}\right)
$$

or $m_{0}<m_{p}=1 / 2\left(\frac{\beta \mathrm{F}_{2}}{k_{m i}}+\frac{S}{Q \mathrm{~F}_{2}}\right), \quad$ where $\quad S=S_{i}+S_{m}$.

In the limit of low e-beam current density, the second term in parentheses is negligible and the condition for stability reduces to that found previously [16]. In its present form the maximum metastable density is a function of e-beam source strength.

The stability criterion as presented in eq. (22) is not very useful in defining a practical operating regime since it involves the steady-state metastable density, $m_{0}$. In order to express this in terms of experimentally definable parameters, we solve the steady-state equations for $m$. From figure 9,

$$
n_{0}=S_{i} /\left(\beta \mathrm{F}_{2}-k_{m i} m_{0}\right) \text {. }
$$

Using this in the equation for $m$, we arrive at a quadratic equation for $m_{0}$ with solutions,

$$
m_{0}=m_{p} \pm \sqrt{m_{p}^{2}-m_{c}^{2}}
$$

where

$$
m_{c}^{2}=\left(S_{m} \beta F_{2}+S_{i} k_{m} N\right) / Q F_{2} k_{m i}
$$

If $m_{c}<m_{p}$, then two real solutions exist, one of which, $m_{0}<m_{p}$, is stable and the other, $m_{0}>m_{p}$, is unstable. The stable solution is the one which is reached first as $m$ increases from zero. Stability will be maintained as long as $m_{c}<m_{p}$ is satisfied.

A new stability criterion can now be written in 
the form of an upper limit on $E / N$ or discharge voltage, i.e.

$k_{m}\left(\frac{E}{N}, \frac{n}{N}\right)<\frac{k_{m i} Q \mathrm{~F}_{2}}{S_{i} N}\left[\frac{1}{4}\left(\frac{\beta \mathrm{F}_{2}}{k_{m i}}+\frac{S}{Q \mathrm{~F}_{2}}\right)^{2}-\frac{S_{m} \beta}{Q k_{m i}}\right]$.

The term on the right side of the inequality is only weakly dependent on $E / N$. The value of $n / N$ on the left is the steady-state fractional ionization at the point where

$$
m_{0}=m_{p} \text { and } n_{0}=2 S_{i} /\left(\beta F_{2}-k_{m i} S / Q F_{2}\right) \text {. }
$$

For a given e-beam current, gas density, $N$, and fluorine density, $F_{2}$, we can use figure 10 and eq. (24) to establish the critical value of $E / N$ above which the discharge current will run away. Experimentally, with the current rise limited by circuit inductance, this runaway is seen as a drop in discharge impedance and a cessation of lasing.

In figure 11 the critical value of $E / N$ is plotted as a function of e-beam power density for several values of total pressure and fluorine concentration. The experimental points are taken from the current and voltage waveforms obtained on the $0.1 l$ device described in reference [1]. The functional dependence of $E / N$ on $P_{e b}$ is roughly $E / N \propto P_{e b}^{-0.5}$ for the conditions indicated. The dependence of $E / N$ on $\mathrm{F}_{2}$ concentration is likewise $E / N \propto \mathrm{F}_{2}^{1.5}$ and on total number density $E / N \propto N^{-0.5}$. These expressions are only approximately valid within the range of parameters given in figure 11 .

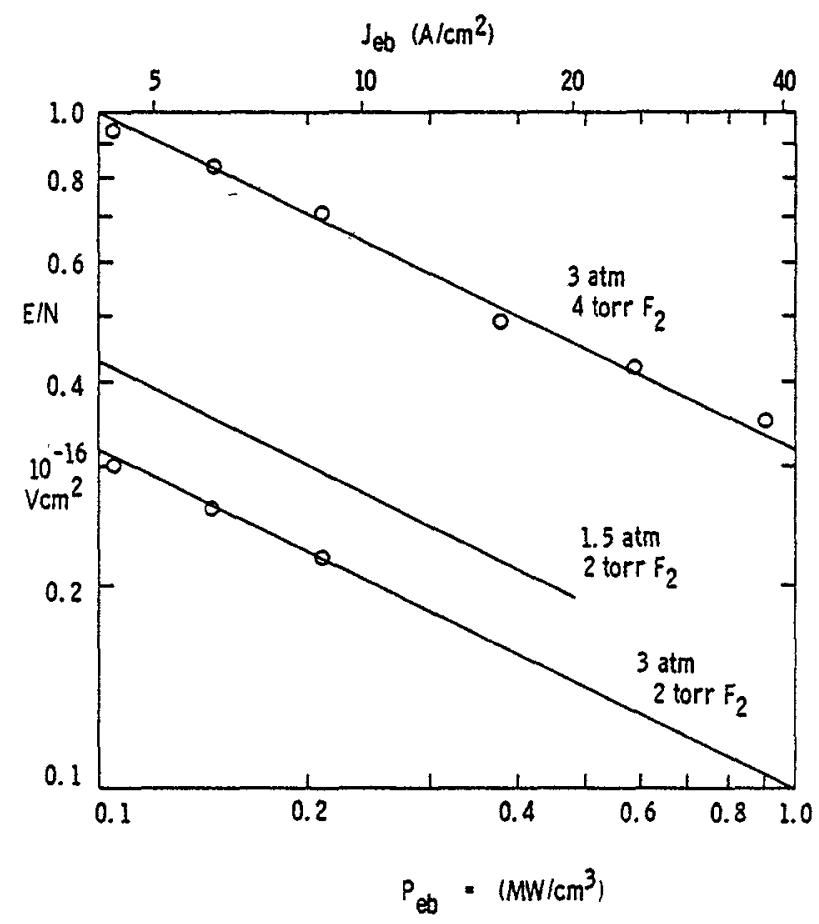

1 19. 11. - Maximum $E / N$ vs. e-beam density.
A subsidiary criterion for stability is evident from the expression for steady-state electron density, i.e.

$$
n_{0}=\frac{S_{i}}{\beta \mathrm{F}_{2}-k_{m i} m_{0}} .
$$

In order for $n_{0}$ to be positive, $m_{0}$ must be less than $\beta \mathrm{F}_{2} / k_{m i}$. If $S / Q \mathrm{~F}_{2}>\beta \mathrm{F}_{2} / k_{m i}$, then eq. (25) becomes the limiting condition on $E / N$. However, over the range of parameters considered here, $S / Q \mathrm{~F}_{2}$ is always less than $\beta \mathrm{F}_{2} / k_{m i}$.

Knowing the value of $E / N$ at which the discharge becomes unstable, we can determine the maximum discharge power loading under given operating conditions. The discharge power density is given by

$$
P_{d}=J . E=e n v_{e} E .
$$

The electron drift velocity, $v_{e}$, was shown in figure 3 as a function of $E / N$ and $n / N$. Within the range,

$$
1 T d<E / N<10 T d \text { and } 10^{-6}<n / N<10^{-5} \text {, }
$$

$v_{e}$ can be approximated by

$$
v_{e}=b\left(\frac{n}{N}\right)^{0.2}\left(\frac{E}{N}\right)^{0.4} \text {. }
$$

Then $P_{d}$ is given by

$$
P_{d}=e b N^{2}\left(\frac{n}{N}\right)^{1.2}\left(\frac{E}{N}\right)^{1.4}
$$

For $m_{0} \ll m_{p}, n / N$ is proportional to $J_{e b} / \mathrm{F}_{2}$. The maximum $E / N$ is proportional to $J_{e b}^{-0.5} \mathrm{~F}_{2}^{1.5} N^{-1.0}$ Therefore $P_{d} \propto J_{e b}^{0.5} \mathrm{~F}_{2}^{0.9} N^{0.6}$. Writing this in terms of the e-beam power density $P_{e b} \sim J_{e b} N$, we have

$$
P_{d} \propto P_{e b}^{0.5} \mathrm{~F}_{2}^{0.9} N^{0.1} .
$$

The maximum discharge power density is proportional to the square root of the e-beam power deposition. This means that the power enhancement factor, $P_{d} / P_{e b}$, is smaller at higher e-beam current densities. It is larger at higher $F_{2}$ concentrations and, in a given mixture, at higher total pressures.

For overall system efficiency, it is desirable to have $P_{d} / P_{e b}$ as large as possible. In this respect then it is better to operate at low beam currents and high $\mathrm{F}_{2}$ and total pressures. The limitation in going to low e-beam current densities is that as the total power is reduced, the ratio of small signal gain to absorption is less and extraction efficiency becomes poor, figure 8 . The limitation on fluorine concentration arises from quenching of the $\mathrm{KrF}^{*}$ state and absorption by $F_{2}$ and $F_{\text {- }}$. At higher pressures there is more three-body quenching of $\mathrm{KrF}^{*}$ and increased formation of the trimers, $\mathrm{Ar}_{2} \mathrm{~F}^{*}, \mathrm{ArKrF}^{*}$ and $\mathrm{Kr}_{2} \mathrm{~F}^{*}$ by interception of energy channels leading to $\mathrm{KrF}^{*}$. There is therefore an optimum choice of the parameters $N, \mathrm{~F}_{2}$, and $J_{e b}$, which will give the highest overall efficiency and laser output. 


\section{Scaling problems of the $\mathrm{KrF}$ laser. - The $\mathrm{KrF}$} laser appears to have the greatest potential of all other candidates for scaling to very high average powers in the near ultraviolet. The highest specific energy $(30 \mathrm{~J} / 1)$ and efficiency $(10 \%)$ to date have been demonstrated in a small device (0.12 1) with an e-beam sustained discharge. In larger devices, however, discharge loading has not been nearly as effective and in most cases has been abandoned as a viable pumping technique. We believe that efficient discharge pumping in large devices can be achieved and that this represents the best alternative for a reasonably sized high average power laser.

We have undertaken a systematic study of the electron kinetics, discharge stability, e-beam deposition, and optical extraction in order to determine the scalability of the $\mathrm{KrF}$ laser and to assess the relative merits of e-beam versus discharge pumping. We find that discharge power loading is limited by the nonuniformity of e-beam deposition and by the ability of the external circuit to couple energy into a low-impedance load. If the energy deposition is made uniform through the use of opposed e-beams and the device is operated at low beam currents to maximize the discharge impedance, then an extracted laser energy of $45 \mathrm{~J} / 1$ may be achieved with $8 \%$ efficiency. This is about a factor of three more energy than could be extracted with an e-beam alone. The implications are that a much smaller device can be built for a given average power with all the associated savings in optics, size and gas handling equipment.

The maximum discharge power loading, $P_{d}$, consistent with stable, long-pulse operation is proportional to the square root of the e-beam power density, $P_{e}$. This relationship has been derived from a discharge stability analysis and has been confirmed experimentally. The stability criterion for discharge pumping is then,

$$
P_{d}<\gamma P_{e}^{1 / 2}
$$

For the gas mixture $94.8 \% \operatorname{Ar~} 5 \% \mathrm{Kr} 0.17 \% \mathrm{~F}_{2}$ at a total pressure of $3 \mathrm{~atm}$, the constant, $\gamma$, is equal to 1.3 when $P_{d}$ and $P_{e}$ are given in $\mathrm{MW} / \mathrm{cm}^{3}$. The above condition must be satisfied at every point in the discharge or two-stage ionization will lead to volume runaway and eventual arcing.

Another condition which must be satisfied in the discharge is current continuity. Near the center of large planar electrodes, this implies constant current density along a field line, i.e.

$$
J_{d}=e n v_{e}\left(\frac{E}{N}, \frac{n}{N}\right)=\mathrm{constant}
$$

where $E$ is the electric field strength and $N$ is the gas density. Since the electron density, $n$, is controlled by the e-beam deposition which varies with distance from the foil, the electric field must compensate by changing the electron drift velocity, $v_{e}$.
Using the expression for $v_{e}$ from eq. (26), the current density is given by,

$$
J_{d}=e N b\left(\frac{n}{N}\right)^{1.2}\left(\frac{E}{N}\right)^{0.4}
$$

from which it is clear that along a field line $E / N$ must vary inversely as the cube of $n / N$ in order to keep the current density constant.

Monte Carlo simulation studies and experimental measurements of the e-beam energy deposition in gases show that the deposition falls off more or less linearly away from the foil for the range of beam energies and foil thicknesses considered here. This spatial dependence can be written as,

$$
P_{e}(x)=P_{e 0}[1+(r-1) x / d]
$$

where $r$ is the ratio of the power density at a distance $d$ from the foil to the power density at the foil. The source density, $S$, for generating electrons in the gas is related to the e-beam power density by $S=P_{e} / W_{i}$, where $W_{i}$ is the energy dissipated in creating an electron-ion pair. (For argon, $W_{i}=26.2 \mathrm{eV}$.) Since the principal loss of electrons is by attachment to fluorine, the electron density is given by,

$$
n=S / \beta \mathrm{F}_{2}=P_{e} / \beta \mathrm{F}_{2} W_{i}
$$

where $\beta$ is the attachment coefficient and $F_{2}$ is the fluorine density.

The discharge power density is given by,

$$
P_{d}=N J_{d} E / N
$$

and since $J_{d}$ is constant along a field line, $P_{d}$ varies directly as $E / N$.

From eqs. (30) and (32) then, we see that $P_{d}$ must vary inversely as the cube of $P_{e}$ (Fig. 12), or

$$
P_{d}(x)=c[1+(r-1) x / d]^{-3} .
$$

The constant $c$ will now be chosen so that the stability criterion, eq. (27) is satisfied throughout the discharge. It is clear from eq. (34) that the condition is most likely to be violated at $x=d$. Therefore, we set $P_{d}(d)=\gamma P_{e}^{1 / 2}(d)$ or $c r^{-3}=\gamma r^{1 / 2} P_{e 0}^{1 / 2}$ so that $c$ is given by $c=\gamma r^{7 / 2} P_{e 0}^{1 / 2}$. The average e-beam and discharge power densities can now be found by integrating eqs. (31) and (34) over $x$ from 0 to $d$. Thus,

$$
\bar{P}_{e}=P_{e 0}(1+r) / 2
$$
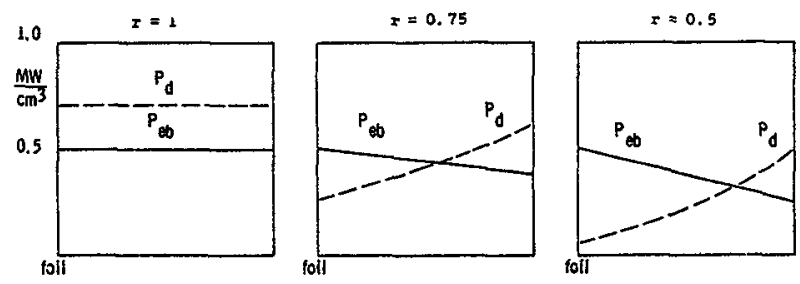

Fig. 12. - Discharge current continuity. 
and

$$
\bar{P}_{d}=\gamma P_{e 0}^{1 / 2} r^{3 / 2}(1+r) / 2 .
$$

If the discharge power is supplied by a simple capacitive discharge circuit, where maximum power is reached at the end of the e-beam pulse, then the discharge power averaged over time is just half of that given in eq. (36). The total energy dissipated in the laser is then,

$$
\varepsilon_{i n}=\left(\bar{P}_{e}+\bar{P}_{d} / 2\right) \tau_{p}
$$

where $\tau_{p}$ is the pulse length.

The quantum efficiency for converting e-beam energy into excited $\mathrm{KrF}$ is $\mathrm{h} v\left(1+\delta_{e}\right) / W_{i}=25 \%$, where $\delta_{e}$ is the fraction of excited states produced for every ion pair. (For argon $\delta_{e}=0.29$.) The quantum efficiency for converting discharge energy into $\mathrm{KrF}^{*}$ states is $36 \%$. The actual conversion efficiencies are somewhat lower due to formation of trimers and the loss of $\mathrm{ArF}^{*}$ to quenching and spontaneous emission. In an optimized gas mixture, conversion efficiencies of $\eta_{e}=20 \%$ and $\eta_{d}=25 \%$ can readily be achieved. These values are independent of input power density. The average production rate of $\mathrm{KrF}^{*}$ per unit volume, $R_{p}$, can now be written,

$$
R_{p}=\left(\eta_{e} P_{e 0}+\eta_{d} \gamma P_{e 0}^{1 / 2} r^{3 / 2} / 2\right)(1+r) / 2 h v .
$$

Since the ground state of $\mathrm{KrF}^{*}$ is dissociative, the small-signal gain is given by $g_{0}=\sigma N^{*}=\sigma R_{p} \tau$, where $\sigma$ is the cross section for stimulated emission and $\tau$ is the total $\mathrm{KrF}^{*}$ lifetime. Another way of writing this relation is $g_{0}=R_{p} h v / I_{s}$, where $I_{s}=h v / \sigma \tau$ is the saturation intensity. Using a spontaneous emission lifetime of 9 ns [17], a stimulated emission cross section of $1.9 \AA$ and the published fluorine and three-body quenching rates [18] of $\mathrm{KrF}^{*}$, the saturation intensity for the mixture $94.8 \% \mathrm{Ar} 5 \% \mathrm{Kr}$ $0.17 \% \mathrm{~F}_{2}$ at $3 \mathrm{~atm}$ is $2.6 \mathrm{MW} / \mathrm{cm}^{2}$. The expression for the average small-signal gain is then,

$$
g_{0}=\left(\eta_{e} P_{e 0}+\eta_{d} \gamma P_{e 0}^{1 / 2} r^{3 / 2}\right)(1+r) / 2 I_{s}
$$

Since the discharge power density is greatest where the e-beam power density is lowest, the sum of the two is fairly constant and the error in using averaged quantities is minimal.

The laser output intensity is given in terms of the small-signal gain, the gain length, $l$, the mirror reflectivity, $R$, and the absorption in the laser medium, $\alpha$, by the expression,

$$
I_{\text {out }}=\frac{I_{s}}{2} \ln \frac{1}{R}\left(\frac{g_{0}}{x+\frac{1}{2 l} \ln \frac{1}{R}}-1\right)
$$

This expression has been compared with an exact numerical solution of the cavity equations, and was found in good agreement for values of $R$ down to $30 \%$.

The maximum output intensity corresponding to complete extraction of all laser photons is

$$
I_{\max }=R_{p} h v V / A=g_{0} l I_{s}
$$

so the extraction efficiency can be defined as,

$$
\eta_{\mathrm{ext}}=\frac{I_{\mathrm{out}}}{I_{\max }}=\frac{1}{2 l} \ln \frac{1}{R}\left(\frac{1}{\alpha+\frac{1}{2 l} \ln \frac{1}{R}}-\frac{1}{g_{0}}\right) .
$$

The optimum reflectivity is found from eq. (42) to be

$$
\frac{1}{2 l} \ln \frac{1}{R}=\sqrt{\alpha g_{0}}-\alpha
$$

which corresponds to a maximum extraction efficiency of

$$
\eta_{\mathrm{ext}}^{\max }=\left(1-\sqrt{\frac{a}{g_{0}}}\right)^{2} .
$$

The extracted laser energy density $\varepsilon_{\text {out }}$ can now be written as

$$
\varepsilon_{\mathrm{out}}=\eta_{\mathrm{ext}} R_{p} h v \tau_{p}
$$

Absorption in the laser medium is made up of two types : 1) static absorption by molecular fluorine and 2) transient absorption due to $\mathrm{F}^{-}$, positive molecular ions, and possibly excited electronic states. Our absorption measurements show that the dominant transient absorption is due to the ions, $\mathrm{F}^{-}, \mathrm{Kr}_{2}^{+}$ and $\mathrm{Ar}_{2}^{+}$. Since the ions are lost by recombination, their population is proportional to the square root of the e-beam power density. We can therefore write,

$$
\alpha=\sqrt{c \bar{P}_{e} / W_{i}}+\sigma_{\mathrm{F}_{2}}\left[\mathrm{~F}_{2}\right]
$$

where $c$ is a constant determined experimentally to be $4 \times 10^{-28} \mathrm{~cm} \mathrm{~s}$ and $\sigma_{\mathrm{F}_{2}}=1.5 \times 10^{-20} \mathrm{~cm}^{2}$.

The e-beam pulse length is chosen so that

$$
\tau_{p} J_{e}=10 \mu \mathrm{C} / \mathrm{cm}^{2} \text {. }
$$

This value has been found to be an upper limit for the consistent operation of a single shot e-beam device without foil damage. This limitation also points out the advantage of operating at the highest pressure consistent with good laser efficiency, since the e-beam energy deposition, $P_{e} \tau_{p}$, is now independent of the current density but proportional to gas density. We have chosen our test case at $3 \mathrm{~atm}$, because this pressure has resulted in efficient laser operation in small devices. The reason that lower pressures have been found to give higher output in large devices is because of poor e-beam penetration at high pressures. The nonuniformity of e-beam 


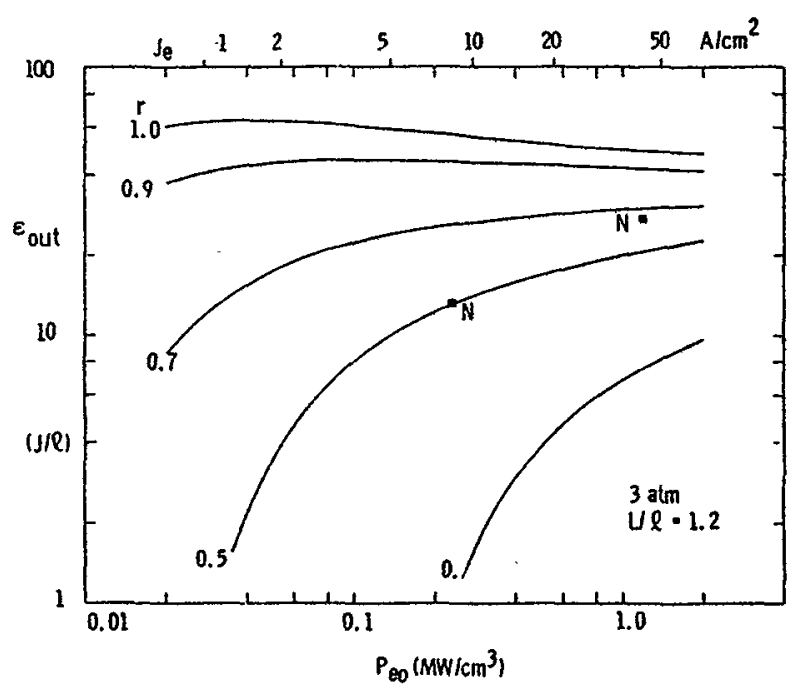

Fig. 13. - Spectfic energy (e-beam + discharge). ( \pm ) Experımental data obtained on two northrop devices.

deposition also limits the discharge energy loading in large devices, as we shall see below.

We now have all the information necessary to determine the extracted laser energy density from eq. (45). This is plotted as a function of $P_{\mathrm{e} 0}$ in figure 13 for the mixture $94.8 \% \operatorname{Ar~} 5 \% \mathrm{Kr} 0.17 \% \mathrm{~F}_{2}$ at $3 \mathrm{~atm}$. The corresponding e-beam current density is given on the upper scale. The curves show that a high extracted laser energy is possible at low e-beam current densities if the e-beam deposition is uniform. The uniformity becomes more critical the smaller the value of $P_{e 0}$. When $r=0$ there is no benefit from discharge pumping at all, since the discharge is always unstable.

The small-signal gain and absorption for the same gas mixture are plotted in figure 14 as a function of e-beam power density. The horizontal line at $0.24 \% \mathrm{~cm}^{-1}$ is the absorption due to 4 torr of fluorine with the factor $L / l=1.2$ taken into account. ( $L$ is

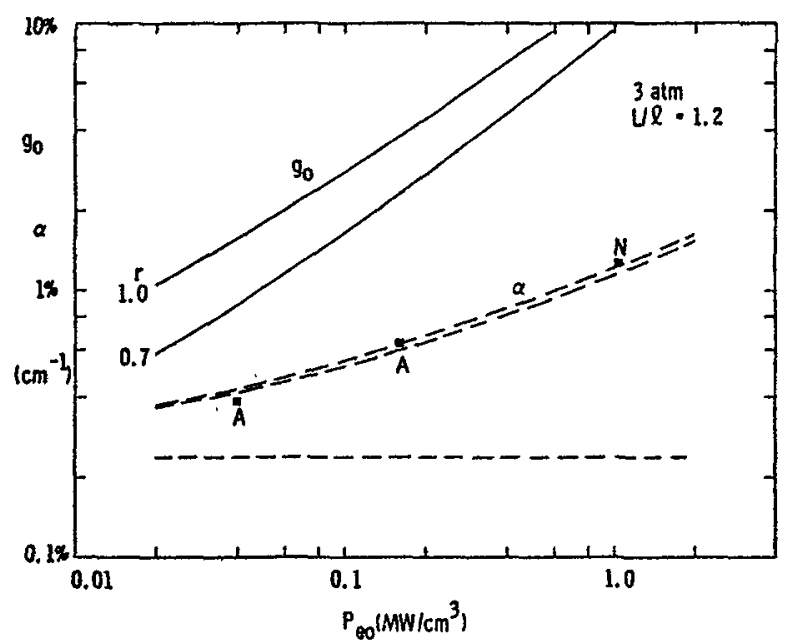

Fig. 14. - Small signal gain and absorption (e-beam + discharge). the distance between the laser mirrors, or windows, which is filled with fluorine. The value 1.2 is required to ensure electrical isolation of the electrodes from the mirror mounts.) Most of the remaining absorption is due to the ions $\mathrm{F}^{-}, \mathrm{Ar}_{2}^{+}$and $\mathrm{Kr}_{2}^{+}$which are proportional to $P_{e 0}^{1 / 2}$. The measurements by AVCO at the same pressure in a slightly different mix are shown for comparison. Their values, $A$, for the transient absorption at 1.5 and $6 \mathrm{~A} / \mathrm{cm}^{2}$ are added to our baseline at $0.24 \% \mathrm{~cm}^{-1}$. The small-signal gain is seen to increase faster than the absorption in going to harder pumping.

From eq. (43) it is seen that, for a given smallsignal gain and absorption, the optimum mirror reflectivity, $R$, is determined by the gain length, $l$. As the laser is increased in length, the optimum value of $R$ is reduced. However, there is a minimum value of $R$ below which the mode quality of the laser suffers due to superfluorescence. The minimum reflectivity is found experimentally to be about $20 \%$. This condition sets a limit on the length of the laser given by,

$$
l^{\max }=\frac{\ln \left(1 / R_{\min }\right)}{2\left(\sqrt{\alpha g_{0}}-\alpha\right)} .
$$

Thus, as the input power density increases and the small-signal gain and absorption go up, the maximum length of the device is reduced. This is an important consideration in scaling to high pulse energies.

In figure 15 the overall laser efficiency, maximum gain length and discharge enhancement factor are plotted as a function of e-beam power density. The discharge enhancement factor is defined as the ratio of energy deposited in the gas by the discharge to that deposited by the e-beam. It can be determined from eq. (35) and (36) as

$$
\bar{P}_{d} / 2 \bar{P}_{e}=\gamma r^{3 / 2} / 2 P_{e 0}^{1 / 2} .
$$

The overall efficiency is an increasing function

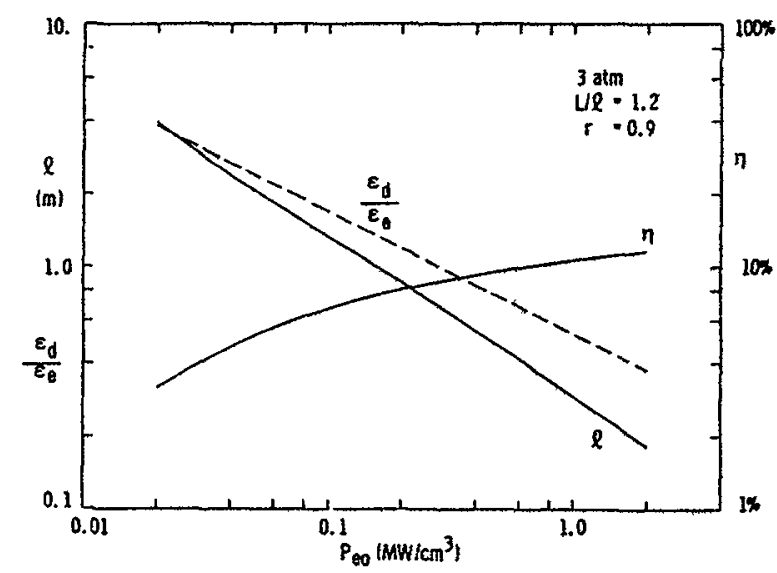

Fig. 15. - Maximum gain length and efficiency (e-beam + discharge). 


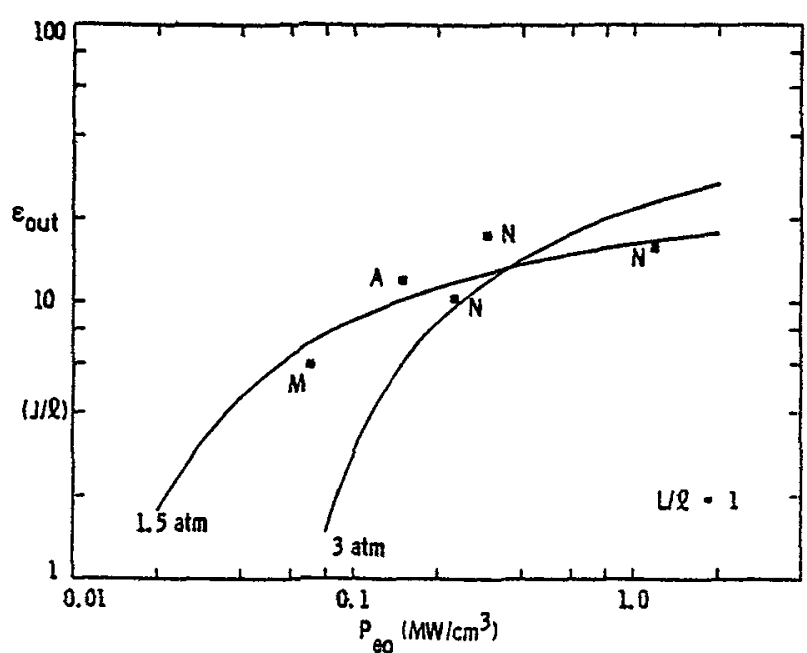

Fig. 16. - Specific energy (e-beam only). $M(\boldsymbol{a})$ Experimental data obtained on Maxwell device.

of $P_{e 0}$ since the small signal gain is increasing faster than the absorption. From this graph we can see that there is a tradeoff to be made between the size of a device and the operating efficiency. With a one meter gain length, an extracted energy of $45 \mathrm{~J} / 1 \mathrm{can}$ be achieved at $8 \%$ efficiency with an e-beam current density of $6 \mathrm{~A} / \mathrm{cm}^{2}$. In contrast, a one meter laser pumped by an e-beam alone at $12 \mathrm{~A} / \mathrm{cm}^{2}$ can deliver only $11 \mathrm{~J} / 1$ at $8 \%$ efficiency (see Fig. 16 and 17 ). The difficulty comes in achieving less than $10 \%$ variation in e-beam deposition in the field direction. We feel that the only way this can be done is with two opposed e-beams.

Some comparison runs with e-beam pumping only were made assuming uniform deposition $(r=1)$ and $L / l=1$. The extracted energy density is plotted in figure 16 for 1.5 and 3 atm total pressure. Two conclusions may be drawn from these curves : 1) harder pumping works best at higher pressures and 2) the extracted energy always increases with pumping power. The best experimental results of Maxwell

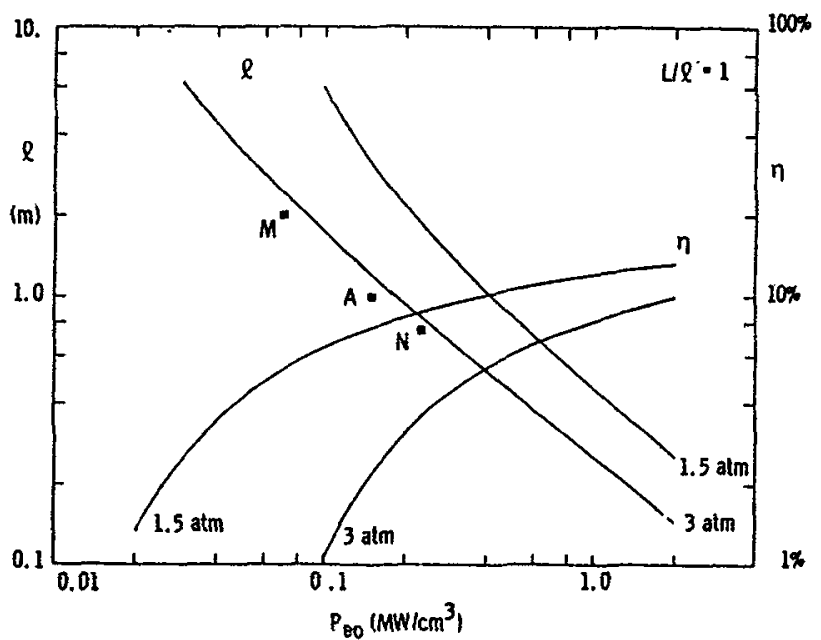

Fig. 17. - Maximum gain length and efficiency (e-beam only).

(2 atm), AVCO (1.7 atm) and Northrop are in good agreement with the predictions.

The maximum gain length and overall efficiency for an e-beam pumped $\mathrm{KrF}^{*}$ laser are shown in figure 17. Again the tradeoff between device size and efficiency is clear. The lasers of Maxwell, AVCO and Northrop are seen to be very close to the limit of scalability. The only way to go to higher than 10 to $12 \mathrm{~J} / 1$ without severely limiting the device size is through combined e-beam and discharge pumping with the uniform deposition achieved by opposing beams.

In conclusion, e-beam sustained discharge pumping seems to have some distinct advantages especially for high average power excimer lasers, even though some additional complexities are involved in discharge pumping. These complexities arise primarily due to nonuniformity of the e-beam deposition and consequent discharge instability. Solution to these problems appears to be feasible using two opposing e-beams to provide uniform ionization.

\section{References}

[1] Bradford, R. S., Jr, Lacina, W. B., Ault, E. R. and BhaUMiK, M. L., Optics Commun. 18 (1976) 210

[2] Judd, O., J. Appl. Phys. 47 (1976) 5297.

[3] Lacina, W. B., Presented at Second Winter Colloquium on Laser-Induced Chemistry, Park City, Utah, 1977.

[4] Long, W. H., Jr, Bailey, W. F. and Garscadden, A., Phys. Rev. $A 13$ (1976) 471.

[5] ShK arofsky, I. P., Johnson, T. W. and Bachynski, M. P., The Particle Kinetics of Plasmas (Addison-Wesley Publishing Company, 1966), p. 283.

[6] Milloy, H. B., Crompton, R. W., Rees, J. A. and Robinson, A. G., in Abstracts of Papers of the 9th ICPEAC, ed. J. S. Risley and R. Geballe (University of Washington Press, Seattle, 1975), vol. 1, p. 457.

[7] Frost, L. S. and Phel Ps, A. V., Phys. Rev. 136 (1964) A1538.

[8] SCHAPER, M. and Scheibner, H., Beit. Plasma Phys. 9 (1969) 45 .

[9] JACOB, J. H. and Mangano, J. A., Appl. Phys. Lett. 29 (1976) 467.
[10] Rapp, D. and Englander-Golden, P., J. Chem. Phys. 43 (1965) 1464.

[11] HaLl, R. J., J. Chem. Phys. 68 (Feb. 1978) 1803.

[12] Chantry, P. J., Westinghouse Rep. 78-9C6-ATACH-R1, March 1978.

[13] Pack, J. L., Voshall, R. E. and Phelps, A. V., Phys. Rev. 127 (1962) 2084

[14] Rober Tson, A. G., in The Diffusion and Drift of Electrons in Gases, L. G. H. Huxley and R. W. Crompton (John Wiley and Sons, New York, 1974), p. 607.

[15] Brambring, J., Z. Phys. (Germany), 179 (1964) 539.

[16] Daugher tY, J. D., Mangano, J. A. and JACOB, J. H., Appl. Phys. Lett. 28 (1976) 581

[17] Burnham, R. and Searles, S. K., J. Chem. Phys. 67 (1977) 5967.

[18] Nakano, H. H., Hill, R. M., LoRenTs, D. C., Huestis, D. L. and MCCuSKer, M. V., SRI Report No. MP 76-99, 1976. 